\title{
INTEGRAL and RXTE observations of accreting millisecond pulsar IGR J00291+5934 in outburst
}

\author{
M. Falanga ${ }^{1,2}$, L. Kuiper ${ }^{3}$, J. Poutanen ${ }^{4}$, E. W. Bonning ${ }^{5}$, W. Hermsen ${ }^{3,6}$, T. Di Salvo ${ }^{7}$, \\ P. Goldoni ${ }^{1,2}$, A. Goldwurm ${ }^{1,2}$, S. E. Shaw ${ }^{8,9}$, and L. Stella ${ }^{10}$ \\ ${ }^{1}$ CEA Saclay, DSM/DAPNIA/Service d'Astrophysique (CNRS FRE 2591), 91191 Gif-sur-Yvette, France \\ e-mail: mfalanga@cea.fr \\ 2 Unité mixte de recherche Astroparticule et Cosmologie, 11 place Berthelot, 75005 Paris, France \\ 3 SRON Netherlands Institute for Space Research, Sorbonnelaan 2, 3584 CA Utrecht, The Netherlands \\ 4 Astronomy Division, PO Box 3000, 90014 University of Oulu, Finland \\ ${ }^{5}$ Laboratoire de l'Univers et de ses Théories, Observatoire de Paris, 92195 Meudon Cedex, France \\ 6 Astronomical Institute “Anton Pannekoek”, University of Amsterdam, Kruislaan 403, 1098 SJ Amsterdam, The Netherlands \\ 7 Dipartimento di Scienze Fisiche ed Astronomiche, Università di Palermo, via Archirafi 36, 90123 Palermo, Italy \\ 8 School of Physics and Astronomy, University of Southampton, SO17 1BJ, UK \\ 9 INTEGRAL Science Data Centre, 1290 Versoix, Switzerland \\ ${ }_{10}$ Osservatorio Astronomico di Roma, via Frascati 33, 00040 Monteporzio Catone, Italy
}

Received 19 May 2005 / Accepted 18 August 2005

\section{ABSTRACT}

\begin{abstract}
Simultaneous observations of the accretion-powered millisecond pulsar IGR J00291+5934 by the International Gamma-Ray Astrophysics Laboratory and Rossi X-ray Timing Explorer during the 2004 December outburst are analysed. The average spectrum is well described by thermal Comptonization with an electron temperature of $50 \mathrm{keV}$ and Thomson optical depth $\tau_{\mathrm{T}} \sim 1$ in a slab geometry. The spectral shape is almost constant during the outburst. For the first time we detect a spin-up, $\dot{v}=8.4 \times 10^{-13} \mathrm{~Hz} \mathrm{~s}^{-1}$, of an accreting millisecond pulsar. The ISGRI data reveal the pulsation of X-rays at a period of 1.67 milliseconds up to $\sim 150 \mathrm{keV}$. The pulsed fraction is shown to increase from 6 per cent at $6 \mathrm{keV}$ to $12-20$ per cent at $100 \mathrm{keV}$. This is naturally explained by the action of the Doppler effect on the exponentially cutoff Comptonization spectrum from the hot spot. The nearly sinusoidal pulses show soft lags with a complex energy dependence, increasing up to $7 \mathrm{keV}$, then decreasing to $15 \mathrm{keV}$, and seemingly saturating at higher energies.
\end{abstract}

Key words. accretion, accretion discs - binaries: close - stars: individual: IGR J00291+5934 - stars: neutron - X-rays: binaries

\section{Introduction}

IGR J00291+5934 was discovered in the galactic disk by the International Gamma-Ray Astrophysics Laboratory (INTEGRAL) (Eckert et al. 2004) in a routine Galactic Plane Scan of the Cassiopeia region on December 2, 2004 during an outburst. Follow-up observations on December 3, 2004 with the Rossi X-ray Timing Explorer (RXTE) classified the source as an accreting X-ray millisecond pulsar (MSP), the sixth known source of this class (Markwardt et al. 2004a). IGR J00291+5934 has a spin period of $1.67 \mathrm{~ms}$, making it the fastest known X-ray MSP to date, and an orbital period of $2.5 \mathrm{~h}$ (Markwardt et al. 2004b). The other known X-ray accreting millisecond pulsars' spin frequencies lie between 180 and $435 \mathrm{~Hz}$ (see review by Wijnands 2005). Their orbital periods fall into two distinct ranges - either around $40 \mathrm{~min}$ or $2-4.5 \mathrm{~h}$.

A candidate optical counterpart was observed with diminishing intensity from magnitude $R \sim 17-21$, consistent with the X-ray flux decay rate of IGR J00291+5934 during the latter days of the outburst (Fox \& Kulkarni 2004; Bikmaev et al. 2004; Steeghs et al. 2004). It is the second X-ray MSP (after SAX J1808.4-3658) for which the optical flux decay could be observed. Spectroscopy of the optical source led to the detection of broad emission lines of HeII and $\mathrm{H} \alpha$ (Roelofs et al. 2004; Filippenko et al. 2004). Radio observations also detected a source consistent with the position of IGR J00291+5934 (Pooley 2004; Fender et al. 2004; Rupen et al. 2004). The source has been observed in quiescence with Chandra by Jonker et al. (2005), and a first INTEGRAL spectrum was reported by Shaw et al. (2005).

The hard X-ray emission of IGR J00291+5934 was significantly detected by INTEGRAL during the entire fifteen-day observation from outburst to quiescence. In this paper we analyze the X-ray spectrum during the outburst. Furthermore, we perform timing analysis on the INTEGRAL high energy data as well as on data from a RXTE follow-up observation, in order to 
Table 1. Log of INTEGRAL observations analysed in this paper. Start and end times are in days of December 2004 (from MJD 53340 ). Exposures are in seconds and count rates are in counts per second, for ISGRI in the 20-100 keV and for JEM-X in the 5-20 keV energy bands.

\begin{tabular}{rrrrrrrl}
\hline \hline & \multicolumn{9}{c}{ ISGRI } & \multicolumn{2}{c}{ JEM-X } \\
Start & End & Exposure & Count rate & Exposure & Count rate & Rev. & Obs. \\
\hline 3.671 & 3.840 & 8449 & $8.3 \pm 0.5$ & 3023 & $4.2 \pm 0.2$ & 261 & Cas A \\
4.717 & 5.437 & 40241 & $7.8 \pm 0.2$ & 10088 & $3.6 \pm 0.1$ & 262 & Cas A \\
5.631 & 6.850 & 63895 & $6.9 \pm 0.2$ & 23911 & $3.2 \pm 0.1$ & 262 & ToO \\
7.689 & 7.801 & 6403 & $4.4 \pm 0.5$ & 5675 & $2.7 \pm 0.2$ & 263 & Cas A \\
7.813 & 9.815 & 117980 & $4.2 \pm 0.1$ & 58679 & $2.3 \pm 0.1$ & 263 & ToO \\
10.710 & 12.835 & 106402 & $1.1 \pm 0.1$ & 70959 & $0.7 \pm 0.1$ & 264 & Cas A \\
13.700 & 15.575 & 94077 & $0.2 \pm 0.1$ & 22281 & $0.1 \pm 0.1$ & 265 & Cas A \\
\hline
\end{tabular}

study the characteristics of the pulse profile (shape, time lags, pulsed fraction) at energies from 2 to $150 \mathrm{keV}$.

\section{Observations and data}

\subsection{INTEGRAL}

The dataset was obtained with INTEGRAL (Winkler et al. 2003) from December 2 to December 16, 2004, i.e. from satellite revolution 261 to 265 (see Table 1). These revolutions include a Target of Opportunity (ToO) observation and part of a Cas A/Tycho AO2 observation.

We use data from the coded mask imager IBIS/ISGRI (Ubertini et al. 2003; Lebrun et al. 2003) at energies from 20 to $\sim 200 \mathrm{keV}$ (total exposure of $437.4 \mathrm{ks}$ ) and from the JEM-X monitor (Lund et al. 2003) at energies 5 to $\sim 20 \mathrm{keV}$ (total exposure time of $194.6 \mathrm{ks})$. For ISGRI, the data were extracted for all pointings with a source position offset $\leq 12^{\circ}$, and for JEM-X with an offset $\leq 3.5^{\circ}$. The spectrometer (SPI) was not used since the source was too weak for the SPI and its $2^{\circ}$ angular resolution is inadequate in this case (see Sect. 3.2). Data reduction was performed using the standard Offline Science Analysis (OSA) version 4.2 distributed by the INTEGRAL Science Data Center (Courvoisier et al. 2003). The algorithms used for the spatial and spectral analyses are described in Goldwurm et al. (2003).

\subsection{RXTE}

After the initial follow-up observations by RXTE starting December 3, 2004, a ToO observation was performed between December 7 and 21 (observation id. 90425), for which the data were made publicly available. For our timing analysis, we also used these data, namely from the two nonimaging X-ray instruments, the Proportional Counter Array (PCA; 2-60 keV) (Jahoda et al. 1996) and the High Energy X-ray Timing Experiment (HEXTE; 15-250 keV) (Rothschild et al. 1998).

The PCA data were collected in the E_125us_64M_0_1s event mode, recording event arrival times with $125 \mu$ s time resolution, and sorting events in 64 PHA channels. Default selection criteria were applied and the final net PCU2 exposure was $105.5 \mathrm{ks}$, for the December 7-10 data. We included in our analysis HEXTE ON-source data, using defaul screening criteria for Cluster 0 and 1 . The screened dead-time corrected HEXTE

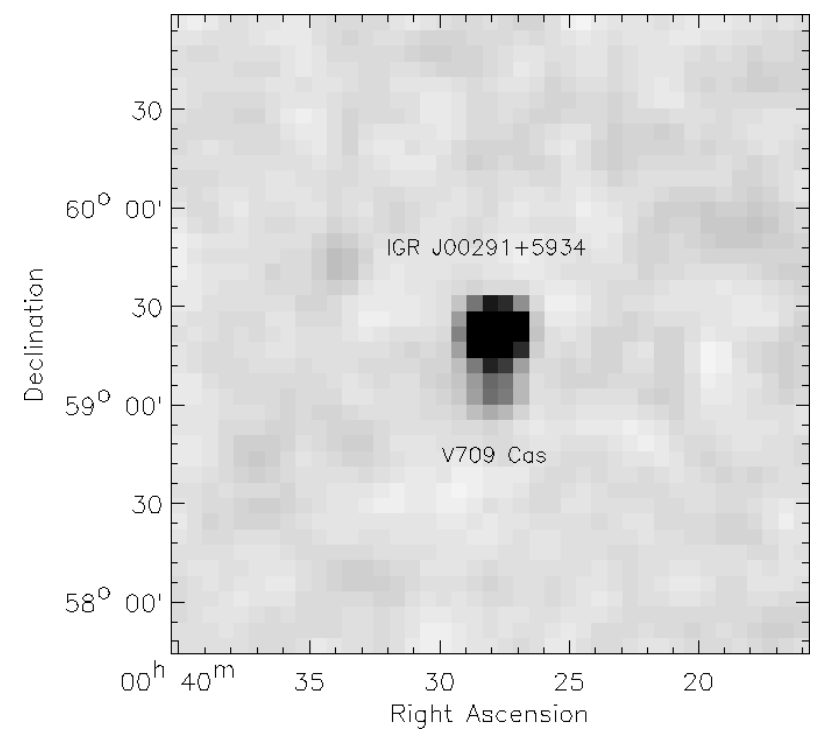

Fig. 1. The 20-40 keV ISGRI mosaicked and deconvolved sky image of the $\sim 437 \mathrm{ks}$ observation. Image size is $\sim 6^{\circ} .25 \times 3^{\circ}$, centered at IGR J00192+5934 position. The pixel size is $5^{\prime}$. IGR J00291+5934 and V709 Cas were detected at a significance of $\sim 88 \sigma$ and $\sim 18 \sigma$, respectively.

Cluster 0 and 1 exposures were $35.7 \mathrm{ks}$ and $36.4 \mathrm{ks}$, respectively, for the December 7-10 data.

\section{Results}

\subsection{IBIS/ISGRI image and light curves}

Figure 1 shows a significance map in the 20-40 keV energy range centered on IGR J00192+5934 located in the Cas A/Tycho region. Single pointings were deconvolved and analyzed separately, and then combined in mosaic images. Two sources are clearly detected at a significance level of $88.4 \sigma$ for IGR J00291 +5934 and $18.2 \sigma$ for the nearby intermediate polar V709 Cas. In the energy band $40-80 \mathrm{keV}$, the significance level was $51.2 \sigma$ for IGR J00291+5934 and $6 \sigma$ for V709 Cas. At the higher energies $80-200 \mathrm{keV}$, the confidence level dropped to $17.1 \sigma$ for IGR J00291 +5934 , while V709 Cas was not detected at a statistically significant level either in single exposures or in the combined image. To obtain precise source locations we simultaneously fitted the ISGRI point spread function to the two close sources. We obtained a 


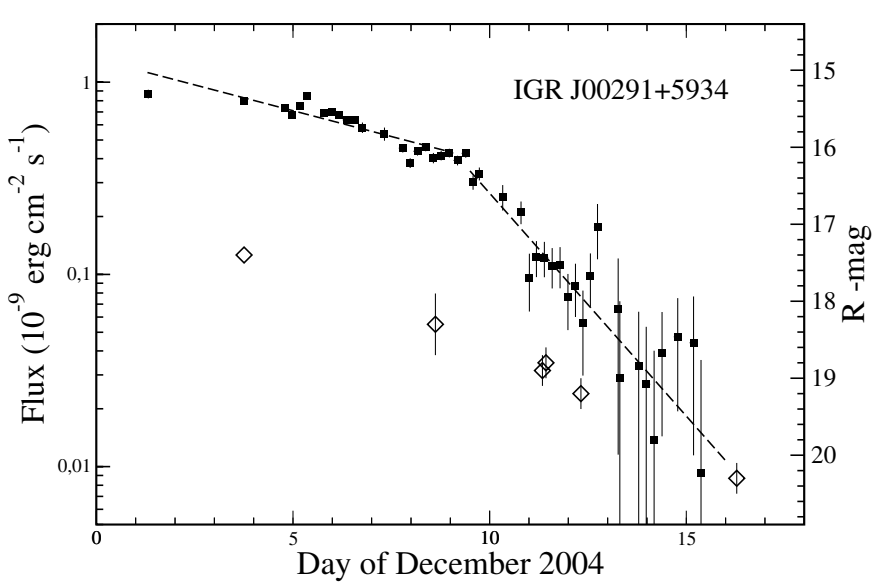

Fig. 2. INTEGRAL/ISGRI light curve in the $20-100 \mathrm{keV}$ energy band (averaged over 0.1 day intervals). The INTEGRAL data from the whole observation (Table 1) have been converted to flux assuming a Comptonization model (see Table 2). The optical flux decay in R-mag is shown with diamonds, where the first two points are taken from Fox \& Kulkarni (2004) and Steeghs et al. (2004), respectively, and the rest was taken from Bikmaev et al. (2004). The dashed lines correspond to $F \propto \mathrm{e}^{-t / 6.6^{\mathrm{d}}}$ and $F \propto \mathrm{e}^{-t / 22^{\mathrm{d}}}$.

position for IGR J00291+5934 at $\alpha_{\mathrm{J} 2000}=00^{\mathrm{h}} 29^{\mathrm{m}} 02^{\mathrm{s}} .92$ and $\delta_{\mathrm{J} 2000}=59^{\circ} 34^{\prime} 06^{\prime \prime} 4$. The position of V709 Cas is given by $\alpha_{\mathrm{J} 2000}=00^{\mathrm{h}} 28^{\mathrm{m}} 55^{\mathrm{s}} .29$ and $\delta_{\mathrm{J} 2000}=59^{\circ} 16^{\prime} 14^{\prime \prime} \cdot 0$. The source position offsets with respect to the optical catalog positions (Fox \& Kulkarni 2004; Downes et al. 1997) are 0'.2 for IGR J00291+5934 and 1'.5 for V709 Cas. The errors are 0'.2 and 1.5 for IGR J00291+5934 and V709 Cas, respectively. These are within the $90 \%$ confidence level assuming the source location error given by Gros et al. (2003). The derived angular distance between the two sources is $\sim 18^{\prime}$. Due to the fact that INTEGRAL is able to image the sky at high angular resolution (12' for ISGRI and $3^{\prime}$ for JEM-X), we were able to clearly distinguish and isolate the high-energy fluxes from the two sources separately. This allowed us to study the X-ray emission of IGR J00291+5934 during its entire outburst.

The INTEGRAL 20-100 keV high energy light curve has been extracted from the images using all available pointings and is shown in Fig. 2, averaged over 0.1 day intervals. The first point corresponds to the detection of the source (Eckert et al. 2004). Using a Comptonization model (see Sect. 3.2.1), we estimated the peak bolometric X-ray flux to be $2.1 \times$ $10^{-9} \mathrm{erg} \mathrm{cm}^{-2} \mathrm{~s}^{-1}$. For a distance of $5 \mathrm{kpc}$ (see Sect. 4.1), this corresponds to a bolometric luminosity of $6.3 \times 10^{36} \mathrm{erg} \mathrm{s}^{-1}$, or $3.5 \%$ of the Eddington luminosity, $L_{\text {Edd }}$, for a $1.4 M_{\odot}$ neutron star. This is a lower value then measured for XTE J1751-305 which has a luminosity of $\sim 0.13 L_{\text {Edd }}$ (Gierliński \& Poutanen 2005), but similar to that of the other accreting X-ray MSPs.

The outburst profile of IGR J00291+5934 (Fig. 2) shows a decay similar to those of four other millisecond pulsars (e.g., Gilfanov et al. 1998; Gierliński \& Poutanen 2005). Only XTE J1807-294 shows a purely exponential decay with a time scale of $\sim 120$ days (Falanga et al. 2005). After the peak the flux declines exponentially, with a decay time-scale of 6.6 days (10 days in SAX J1808.4-3658, 7.2 days for XTE J1751-305), until it reaches a break, after which the flux drops suddenly with a decay time-scale of 2.2 days (1.3 days in SAX J1808.4-3658, 0.6 days for XTE J1751-305). The higher energy (ISGRI) decay time scale is fully consistent with the JEM-X time scale at lower energy (3-20 keV). The decay of the optical flux, also shown in Fig. 2, is similar to that of the $\mathrm{X}$-ray light curve, but somewhat smoother, with a decay timescale of 4.4 days. Note that the optical source was visible up to 45 days after the discovery. The ISGRI light curve was also extracted in the following energy ranges: $20-40 \mathrm{keV}, 40-80 \mathrm{keV}$ and $80-200 \mathrm{keV}$. The hardness ratio of these energy bands as a function of time indicates that no significant spectral variability was detected.

\subsection{Spectral analysis}

We verified that during the whole observation V709 Cas was observed up to $80 \mathrm{keV}$ with a constant mean count rate of $\sim 0.6$ in the $20-80 \mathrm{keV}$ energy band, and does not influence our spectral analysis for IGR J00291+5934. We performed the spectral analysis using XSPEC version 11.3 (Arnaud 1996), combining the 20-200 keV ISGRI data with the simultaneous 5-20 keV JEM-X data. A constant factor was included in the fit to take into account the uncertainty in the cross-calibration of the instruments. A systematic error of $2 \%$ was applied to the JEM-X/ISGRI spectra which corresponds to the current uncertainty in the response matrix. All spectral uncertainties in the results are given at a $90 \%$ confidence level for a single parameter $\left(\Delta \chi^{2}=2.71\right)$.

\subsubsection{Total spectrum}

The joint JEM-X/ISGRI (5-200 keV) spectrum was first fitted with a simple model consisting of a photoelectrically-absorbed power-law (PL). Given that we were not able to constrain the $N_{\mathrm{H}}$ value (as the JEM-X bandpass starts above $5 \mathrm{keV}$ ) we fixed it to the value found from Chandra observations at lower energies (Nowak et al. 2004). A simple PL model is found to be inadequate with $\chi^{2} /$ d.o.f. $=105 / 36$. The addition of a cut-off significantly improves the fit to $\chi^{2} /$ d.o.f. $=47 / 35$, with the bestfit photon index of $\sim 1.8$ (similar to that reported by Shaw et al. 2005 ) and a cut-off energy of $\sim 130 \mathrm{keV}$. However, this model does not describe the spectrum well below $10 \mathrm{keV}$, which is more complex.

The emission from the accretion shock on a neutron star is expected to be produced by thermal Comptonization of soft seed photons from the star (e.g. Zeldovich \& Shakura 1969; Alme \& Wilson 1973; Lyubarskii \& Sunyaev 1982). Indeed, spectra from accretion-powered MSPs are well described by this model (Gierliński et al. 2002; Poutanen \& Gierliński 2003; Gierliński \& Poutanen 2005; Falanga et al. 2005).

We model the shock emission by the COMPPS model ${ }^{1}$ (Poutanen \& Svensson 1996), where the exact numerical solution of the Comptonization problem in different geometries is obtained by considering successive scattering orders. We approximate the accretion shock geometry by a plane-parallel

\footnotetext{
1 COMPPS is available on

ftp: //ftp.astro.su.se/pub/juri/XSPEC/COMPPS
} 
Table 2. Spectral parameters of the thermal Comptonization COMPPS fit to the JEM-X/ISGRI data.

\begin{tabular}{ll}
\hline \hline$N_{\mathrm{H}}\left(10^{22} \mathrm{~cm}^{-2}\right)$ & 0.28 (f) \\
$k T_{\mathrm{e}}(\mathrm{keV})$ & $49_{-6}^{+2}$ \\
$k T_{\text {seed }}(\mathrm{keV})$ & $1.49_{-0.32}^{+0.16}$ \\
$\tau_{\mathrm{T}}$ & $1.12_{-0.07}^{+0.04}$ \\
$A_{\text {seed }}^{a}\left(\mathrm{~km}^{2}\right)$ & $20.7_{-4.5}^{+12.6}$ \\
$\cos \theta$ & $0.60_{-0.09}^{+0.06}$ \\
$\chi^{2} /$ d.o.f. & $44 / 37$ \\
$L_{1-300 \mathrm{keV}}^{a}\left(10^{36} \mathrm{erg} \mathrm{s}^{-1}\right)$ & 3.7 \\
${ }^{a}$ Assuming a distance of $5 \mathrm{kpc}$.
\end{tabular}

slab at the neutron star surface. The main model parameters are the Thomson optical depth $\tau_{\mathrm{T}}$ across the slab, the electron temperature $T_{\mathrm{e}}$, and the soft seed photon temperature $T_{\text {seed }}$. The emitted spectrum depends also on the angle between the normal and the line of sight $\theta$ which does not coincide with the inclination of the system because of light bending. The seed photons are injected from the bottom of the slab. A fraction $\exp \left(-\tau_{\mathrm{T}} / \cos \theta\right)$ of these photons reaches the observer directly, while the remaining part is scattered in the hot gas. Thus, the total spectrum contains unscattered black body photons and a hard Comptonized tail. The best fit with $\chi^{2} /$ d.o.f. $=44 / 37$ is then obtained for $k T_{\text {seed }} \approx 1.5 \mathrm{keV}, k T_{\mathrm{e}} \approx 50 \mathrm{keV}, \tau_{\mathrm{T}} \approx 1.1$, and $\cos \theta \approx 0.6$ (see Table 2). The model also allows us to determine the apparent area of the seed photons, which turns out to be $A_{\text {seed }} \sim 21(D / 5 \mathrm{kpc})^{2} \mathrm{~km}^{2}$ in the best fit. This corresponds to a hot spot radius of $\sim 2.5 \mathrm{~km}$ in this outburst phase. The unabsorbed $E F_{E}$ spectrum and the residuals of the data to the model are shown in Fig. 3.

Soft emission (presumably from the accretion disk) with a peak temperature of about $0.5 \mathrm{keV}$ has been found in other accreting X-ray MSPs (see e.g. Gierliński \& Poutanen 2005; Falanga et al. 2005) using XMM-Newton data in the $0.5-10 \mathrm{keV}$ energy band. As the JEM-X bandpass begins at $5 \mathrm{keV}$, it is impossible to search for this emission in these data. Neither the $6.4 \mathrm{keV}$ iron line nor Compton reflection were significantly detected.

\subsubsection{Outburst spectrum}

We analysed the one day averaged JEM-X/ISGRI spectra for the observation during the outburst (see Table 1). We plot the best fit results using the thermal Comptonization COMPPS model in Fig. 4. The inclination angle, $\theta$, and the seed photon temperature, $T_{\text {seed }}$, were fixed at the best found fit value for the entire observation (see Table 2 ). The luminosity $L_{\text {bol }}$ was calculated for a distance of $5 \mathrm{kpc}$ from the best fit model in the energy range 1-300 keV.

The results show that the decay of the outburst is marked by a nearly constant plasma temperature, except for the last day of outburst where it shows a decrease along with an increase of the scattering optical depth. Neither variation, however, is very significant statistically.

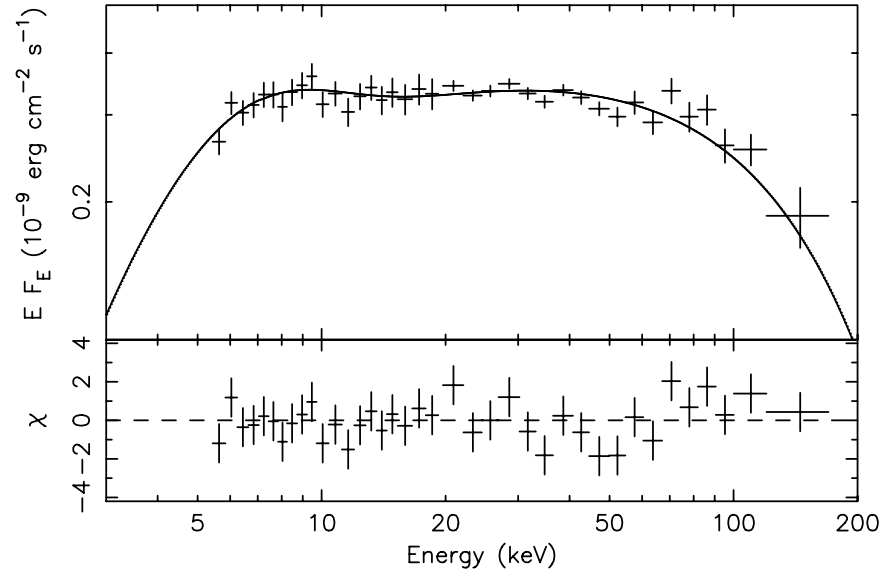

Fig. 3. The unfolded spectrum of IGR J00291+5934 fitted with an absorbed COMPPS model. The data points correspond to the JEM-X (5-20 keV) and ISGRI (20-200 keV) spectra, respectively. The total spectrum of the model is shown by a solid curve. The lower panel presents the residuals between the data and the model.

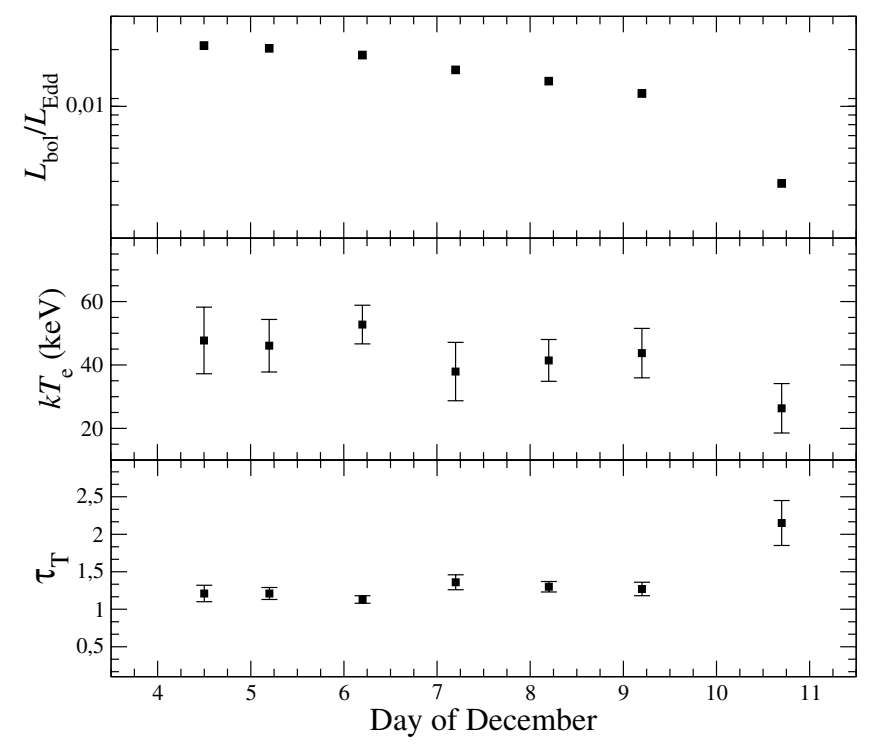

Fig. 4. The outburst evolution of the best-fit spectral parameters of the COMPPS model. Each point, except the last, corresponds to a one day averaged spectrum; due to the lower flux level the last points are averaged over two days.

\subsubsection{Total fluence}

From the broadband spectral model we estimated a total fluence from December 2-15 in the $0.1-300 \mathrm{keV}$ energy band to be $1.37 \times 10^{-3} \mathrm{erg} \mathrm{cm}^{-2}$. The fluence estimated with $R X T E$ is higher by $\sim 0.4 \times 10^{-3} \mathrm{erg} \mathrm{cm}^{-2}$ (Galloway et al. 2005). Owing to the $1^{\circ}$ field of view of $R X T E$, the flux difference could be due to the intermediate polar V709 Cas, located $20^{\prime}$ from IGR J00291+5934. The RXTE pointings were stable around $\alpha_{\mathrm{J} 2000}=00^{\mathrm{h}} 29^{\mathrm{m}} 09^{\mathrm{s}} .23$ and $\delta_{\mathrm{J} 2000}=59^{\circ} 33^{\prime} 58^{\prime \prime}$. 1 , i.e. at every pointing both sources were in the RXTE field of view. We analysed the spectrum of V709 Cas with the identical JEM-X and ISGRI data and exposure times reported in Table 1. The source was detected from 5 to $80 \mathrm{keV}$. In order to estimate the bolometric flux during the outburst period, we fitted the spectrum 
of V709 Cas up to $80 \mathrm{keV}$ with a thermal bremsstrahlung model. The best fit with plasma temperature $\sim 20 \mathrm{keV}$ gives a $\chi^{2} /$ d.o.f. $=12 / 15$. The flux in the energy band $0.1-100 \mathrm{keV}$ was found to be $2.1 \times 10^{-10} \mathrm{erg} \mathrm{cm}^{-2} \mathrm{~s}^{-1}$. The flux of V709 Cas was constant throughout the IGR J00291+5934 outburst, so the total fluence contributed by V709 Cas from December 2 to December 16 was $\sim 0.3 \times 10^{-3} \mathrm{erg} \mathrm{cm}^{-2}$. The difference between our measured total fluence and that of RXTE can therefore be attributed in large part to the flux of V709 Cas. The remainder is likely due to differences in the models used to estimate the source flux and uncertainties in the cross-calibration of the instruments.

\subsection{Timing characteristics}

\subsubsection{The pulsar ephemeris}

Searching the low-statistics ISGRI $(E>20 \mathrm{keV})$ frequency space for the $1.67 \mathrm{~ms}$ signal from IGR J00291+5934 involves making numerous trials, which reduce substantially the sensitivity to periodic signals. Therefore, we have used the highstatistics PCA data (2-60 keV), in order to obtain an accurate ephemeris for the millisecond pulsar.

We first determined the orbital parameters by deriving the instantaneous pulse frequency during sufficiently short exposures (as compared to the orbital period) of typically $200 \mathrm{~s}$, in which the pulse profile could still be significantly recognized. We applied the $Z_{1}^{2}$-statistic (Buccheri et al. 1983) to pulse phase distributions for trial frequencies in a small window centered on the expected pulse frequency. The PCA data until 2004 December 11 (included) yielded sufficiently high signals for $\sim 200 \mathrm{~s}$ integration times. The derived distribution of frequency and frequency uncertainty versus barycentered time (mid of integration interval) was subsequently subjected to an epoch folding method yielding the "best", optimized orbital period $P_{\text {orb}}$. Folding with this best period resulted in an orbital phase versus frequency distribution, which is highly sinusoidal without any significant asymmetry. The amplitude resulting from a sine/cosine fit gave the value of the projected semi-major axis of the neutron star orbit $a_{x} \sin i$, and the minimum of the fit yielded the time of the ascending node $\left(T_{\text {asc }}\right)$.

The derived orbital characteristics based on the PCA data from December 7-11 are fully consistent with those reported by Galloway et al. (2005) based on December 3-6 data. Therefore, in the further analysis we will use the set of orbital parameters derived by Galloway et al. (2005) in order to facilitate direct comparisons (note $T_{\text {asc }}=T_{\pi / 2}-0.25 \cdot P_{\text {orb }}=$ 53345.1619261 MJD TDB).

At this point, we corrected the barycentered time tags of the PCA events for acceleration effects along the orbit. For each PCA sub-observation we determined the time of arrival (TOA) by a pulse profile template correlation analysis similar to the technique normally used in radio-pulsar studies (see e.g. Tempo ${ }^{2}$, Taylor \& Weisberg 1989). The template arrival times could be determined accurately only for PCA data collected between 7 and 12 December 2004

\footnotetext{
${ }^{2}$ http://pulsar.princeton.edu/tempo
}

Table 3. Ephemeris of IGR J00291+5934.

\begin{tabular}{ll}
\hline \hline Parameter & Value \\
\hline Right Ascension (J2000) $^{\dagger}$ & $00^{\mathrm{h}} 29^{\mathrm{m}} 3.0822$ \\
${\text { Declination }(J 2000)^{\dagger}}^{\dagger}$ & $59^{\circ} 34^{\prime} 18^{\prime \prime} .99$ \\
Epoch validity start/end (MJD) & $53341-53353$ \\
Frequency & $598.89213060(1) \mathrm{Hz}$ \\
Frequency derivative & $+8.4(6) \times 10^{-13} \mathrm{~Hz} \mathrm{~s}^{-1}$ \\
Epoch of the period (MJD;TDB) & 53346 \\
Orbital period & $8844.092 \mathrm{~s}$ \\
$a_{x} \sin i$ & $64.993(\mathrm{lt}-\mathrm{ms})$ \\
Eccentricity & 0 \\
Longitude of periastron & $0^{\circ}$ \\
Time of ascending node (MJD;TDB) & 53345.1619261 \\
\hline
\end{tabular}

$\dagger$ The position is based on radio observations with the VLA by Rupen et al. (2004).

(MJD 53 346.202-53351.967), when IGR J00291+5934 was sufficiently strong. Phase folding the template arrival times through a timing model with a constant frequency $v=$ 598.89213064(1) Hz, as found by Galloway et al. (2005), gave rise to large systematic deviations up to 0.15 in phase from the predicted arrival times. A timing model with 2 parameters, $v$ and $\dot{v}$, removed these deviations (all the residuals, TOAs minus model, were $<0.025$ in phase). The fitted timing model has: $v=598.89213060(1) \mathrm{Hz} ; \dot{v}=+8.4(6) \times 10^{-13} \mathrm{~Hz} \mathrm{~s}^{-1}$ at epoch MJD 53346 (TDB). Note that we measure a significant spin-up during this outburst. An error in the assumed source coordinates can give rise to timing errors which may introduce a spurious spin-up or spin-down (Manchester \& Taylor 1977). The average 0.092 arcsec source position error given by Rupen et al. (2004) would introduce a non-existant spin-up rate of $\dot{v}=5.8 \times 10^{-14} \mathrm{~Hz} \mathrm{~s}^{-1}$ during our observation. Since this is smaller than our measured frequency derivative, we conclude that the source was spinning up during the outburst. An apparent spin-up on the order of our measured $\dot{v}$ would require a fairly large $\sim 0.7$ arcsec source position error during our observation. The ephemeris and orbital parameters are given in Table 3.

\subsubsection{INTEGRAL/RXTE pulse profiles and time lags}

We corrected the time stamps of the PCA events from the 2004 December 7-10 (MJD 53346.185 - 53 350.015) observations to arrival times at the solar system barycenter using the JPL DE200 solar system ephemeris and the position of IGR J00291+5934 given in Table 3, taking into account the binary nature of the system. The barycentered times were phase-folded using the pulsar ephemeris given in Table 3. This yielded pulse phase distributions in 64 energy channels. Pulsed emission was detected up to $\sim 60 \mathrm{keV}$. The non-uniformity significance derived by applying a $Z_{1}^{2}$-statistic is even $5.7 \sigma$ for the $34.7-58.1 \mathrm{keV}$ band. The pulse profile shapes remain highly sinusoidal over the $\sim 2-60 \mathrm{keV}$ PCA range (see Fig. 5 panels a-e).

The ISGRI data from INTEGRAL revolutions 261-263 (see Table 1) were used in our timing analysis aiming at the 


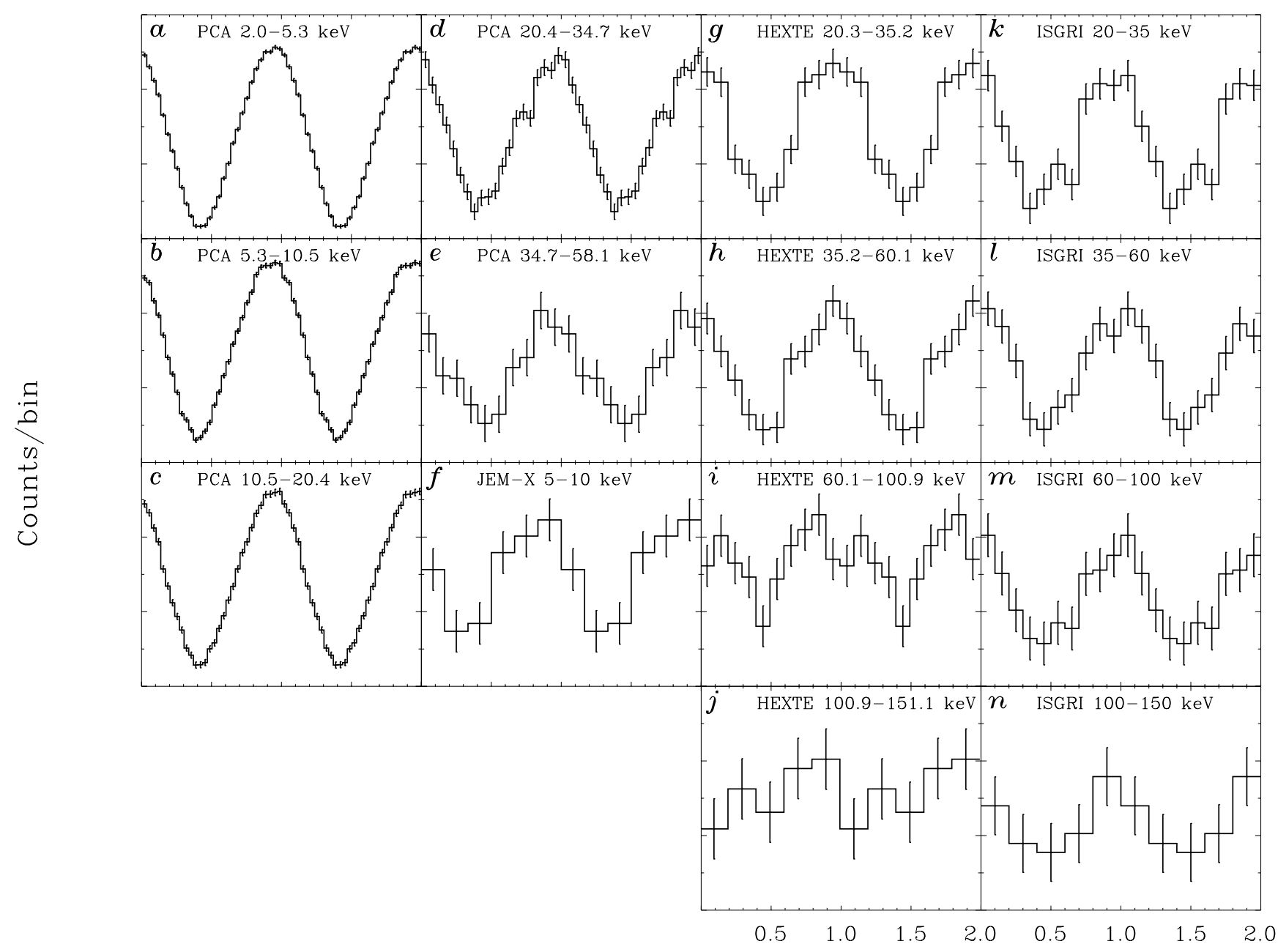

\section{Pulse Phase}

Fig. 5. Pulse-profile collage of IGR J00291+5934 using data from RXTE/PCA (2-58 keV; panels a)-e)), RXTE/HEXTE (20-151 keV; panels g)-j)), INTEGRAL/JEM-X (5-10 keV; panel f)) and INTEGRAL/ISGRI (20-150 keV; panels k)-n)). Two cycles are shown for clarity. All profiles have high off-set values due to the nature of the instruments. The error bars represent 1 sigma statistical errors. All profiles reach their maximum near phase $\sim 0.95$. Notice the highly sinusoidal shape of the profiles for energies up to $100 \mathrm{keV}$.

detection of the 1.67-millisecond timing signal. INTEGRAL science windows ( $\mathrm{scw}$ ) showing erratic (ISGRI) count rate variations, indicative of effects due to Earth radiation belt passage or solar flare activity, were excluded from the analysis. Only time stamps of events with rise times between 7 and 90 channels (Lebrun et al. 2003), detected in non-noisy ISGRI pixels which have an illumination factor of more than $25 \%$ were passed for further analysis. The event times of these selected events are barycentered, and the subsequent pulse phase folding of the barycentered event times using $v, \dot{v}$ and the epoch of the period (see Table 3 ) yielded pulse phase distributions for different energy bands between 20 and $300 \mathrm{keV}$.

Significant pulsed emission was detected up to $100 \mathrm{keV}$, with some indication for a signal also between 100 and $150 \mathrm{keV}$. The $Z_{1}^{2}$ significances for deviations from uniformity were: $9.1 \sigma, 7.3 \sigma, 5.0 \sigma$ and $2.0 \sigma$ for the $20-35,35-60,60-$ 100 and 100-150 keV energy bands, respectively. The sinusoidally shaped pulse profiles are shown in the panels $\mathrm{k}-\mathrm{n}$ of Fig. 5. The $1.67 \mathrm{~ms}$ timing signal was also detected in data from the JEM-X detector: $4 \sigma$ non-uniformity significance for the 5-10 keV band (no event selections except on energy were applied; see Fig. 5 panel f).

To complement and verify our ISGRI timing results we also produced pulse phase distributions based on HEXTE $(15-250 \mathrm{keV})$ data from the same time period as the PCA events above. The HEXTE profiles are shown in panels $\mathrm{g}-\mathrm{j}$ of Fig. 5. The $Z_{1}^{2}$ significances were: $11.4 \sigma, 8.3 \sigma, 3.3 \sigma$ and $1.1 \sigma$ (=no detection) for the energy bands, 20.3-35.2, 35.2-60.1, 60.1-100.9 and $100.9-151.1 \mathrm{keV}$, respectively.

Note that the pulse profiles detected by the PCA, JEM-X, HEXTE and ISGRI are fully consistent in shape and absolute timing. Galloway et al. (2005) reported an energy-dependent time delay, with the 6-9 keV pulses arriving up to $85 \mu$ s earlier than those at lower energies (see Fig. 2 bottom panel of Galloway et al. 2005). Exploiting the ISGRI and HEXTE pulse phase distributions above $20 \mathrm{keV}$, we can investigate this behavior up to $\sim 100 \mathrm{keV}$. We performed a similar analysis, first determining the time lags between profiles in different PCA 


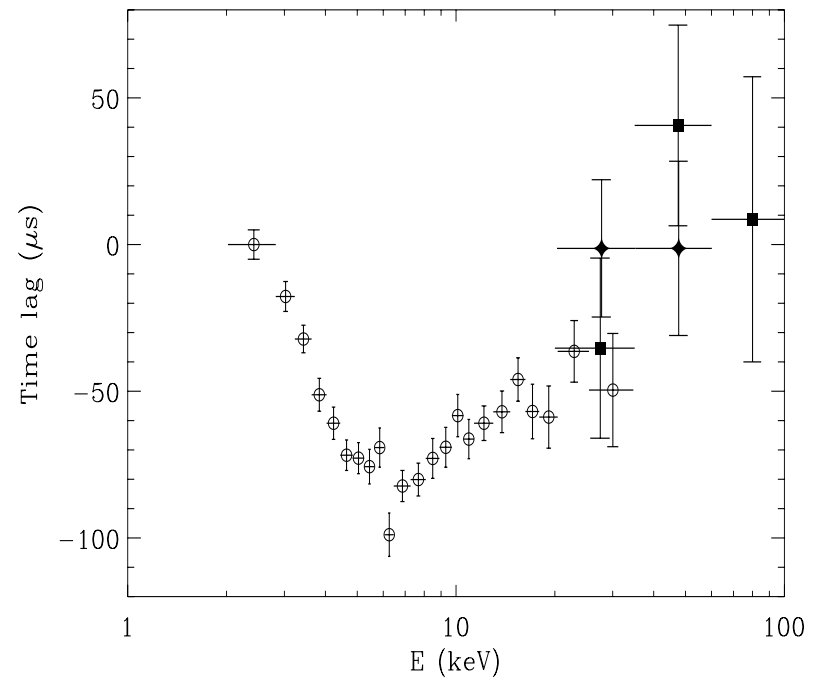

Fig. 6. Time lags as a function of energy in the $2-100 \mathrm{keV}$ energy range combining RXTE/PCA (2-35 keV; 2004 December 7-10; open circles), RXTE/HEXTE (20-60 keV; 2004 December 7-10; filled diamonds) and INTEGRAL/ISGRI (20-100 keV; December 3.6-9.8; filled squares) measurements.

energy bands, but now for the independent PCA dataset from 2004 December 7-10. We confirm the reported variation in time lag between $\sim 2-20 \mathrm{keV}$ as a function of energy (Galloway et al. 2005) and extend the PCA coverage up to $\sim 35 \mathrm{keV}$.

Correlating the three significant ISGRI and two significant HEXTE pulse profiles between $20 \mathrm{keV}$ and $100 \mathrm{keV}$ with the low energy (reference) PCA profile $(2.02-2.82 \mathrm{keV})$, yielded the time lags as measured with ISGRI and HEXTE above $20 \mathrm{keV}$. Note that a time shift of $+0.5 \times 125 \mu$ s has been applied to the PCA time stamps, because the PCA times refer to the start of the time pixel instead of the mid in the case of the E_125us_64M_0_1s event mode. Both the PCA/HEXTE and ISGRI time lags as a function of energy are shown in Fig. 6, covering the $2-100 \mathrm{keV}$ energy band. We see that the ISGRI and PCA/HEXTE time lag measurements in the overlapping $20-35 \mathrm{keV}$ energy band are fully consistent, and that the time lags decrease for increasing energies, attaining values consistent with zero for energies in excess of $35 \mathrm{keV}$ consistent with a trend already set in near $\sim 6 \mathrm{keV}$. The full consistency of the PCA/HEXTE and ISGRI time lags in the overlapping 20-35 keV band indicates that the time alignment between $R X T E$ and INTEGRAL is better than $\sim 25 \mu \mathrm{s}$.

\subsubsection{Spectrum of the pulsed emission and pulsed fraction}

Important diagnostic parameters for constraining the parameter space in theoretical modelling (see e.g. Viironen \& Poutanen 2004) are the pulsed spectrum and the pulsed fraction (here defined as: pulsed flux/total flux) as a function of energy. While the pulsed fluxes can be derived for all the main instruments considered in this study, the total flux of IGR J00291+5934 can only be derived reliably for the high-energy instruments with arcminute imaging capabilities. In particular, the nearby source V709 Cas contaminates the total flux estimate of

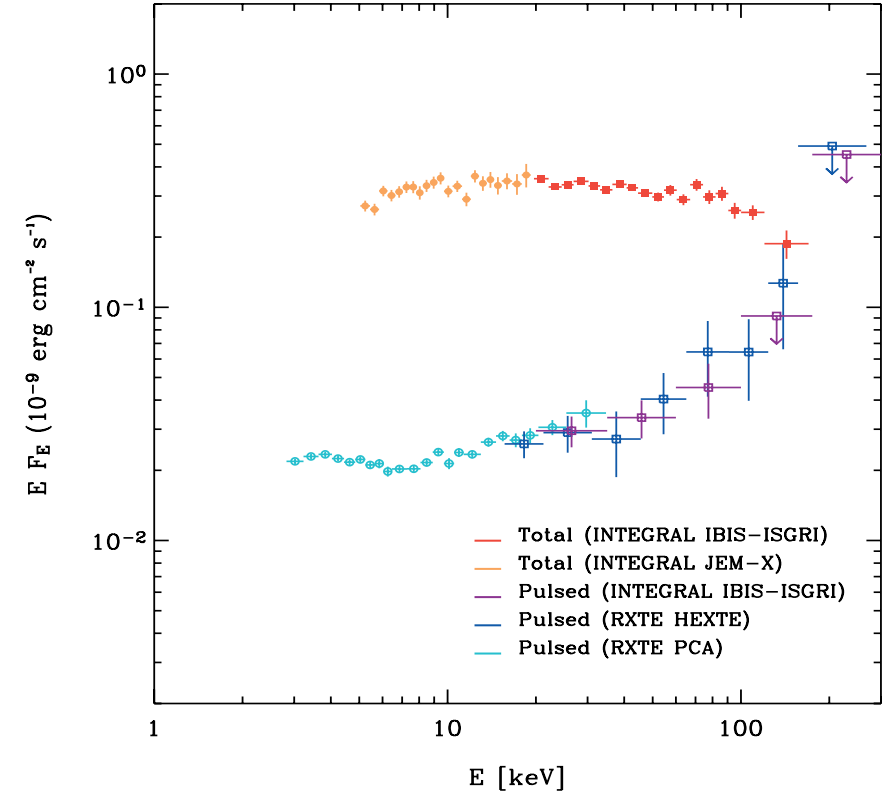

Fig. 7. The total and pulsed spectrum of IGR J00291+5934 in an $E F_{\mathrm{E}}$ representation from 3 to $300 \mathrm{keV}$ combining flux measurements from RXTE/PCA/HEXTE and INTEGRAL/ISGRI for the pulsed part and INTEGRAL/ISGRI and JEM-X for the total part. Notice the hardening of the pulsed spectrum towards higher energies.

IGR J00291+5934 using the non-imaging RXTE instruments (see Sect. 3.2.1). Galloway et al. (2005) did use the PCA to derive the fractional rms amplitude of the pulsations as a function of energy (in their Fig. 2, top panel); therefore, their values contain a contribution from V709 Cas and cannot be used to estimates the fractional rms amplitude of IGR J00291+5934.

We derived the pulsed fluxes for the PCA, HEXTE and ISGRI instruments (the JEM-X statistics for the pulsed component is too low, cf. Fig. 5 panel f) and the total fluxes only for the imaging INTEGRAL instruments ${ }^{3}$. To derive the pulsed fluxes we determined the number of pulsed excess counts in a given energy band. A truncated Fourier-series fit using 3 harmonics to the pulsed phase distributions as a function of energy yielded this quantity. In case of the PCA the pulsed excess counts $(2-30 \mathrm{keV})$ have been converted to flux values $\left(\mathrm{ph} / \mathrm{cm}^{2} \mathrm{~s} \mathrm{keV}\right)$ in a forward energy spectral folding method assuming an underlying absorbed $\left(N_{\mathrm{H}}=2.8 \times 10^{21} \mathrm{~cm}^{-2}\right.$; see Nowak et al. 2004) PL model with an energy dependent index taking into account the different exposures and energy response of the 5 different PCUs. Figure 7 shows these PCA pulsed flux measurements in an $E F_{\mathrm{E}}$ representation.

For HEXTE an equivalent method - dividing the pulsed excess count rates in a certain energy band by its effective sensitive area assuming a PL model - was used to obtain the pulsed fluxes in the 15.6-267.5 keV energy range taking into account the different cluster 0 and 1 dead time corrected $O N$ exposures and energy responses (see Fig. 7).

Finally, for ISGRI the pulsed excess counts (20-300 keV), accumulated during an exposure of $336.94 \mathrm{ks}$, have been

${ }^{3}$ The RXTE and INTEGRAL measurements were nearly contemporaneous. 


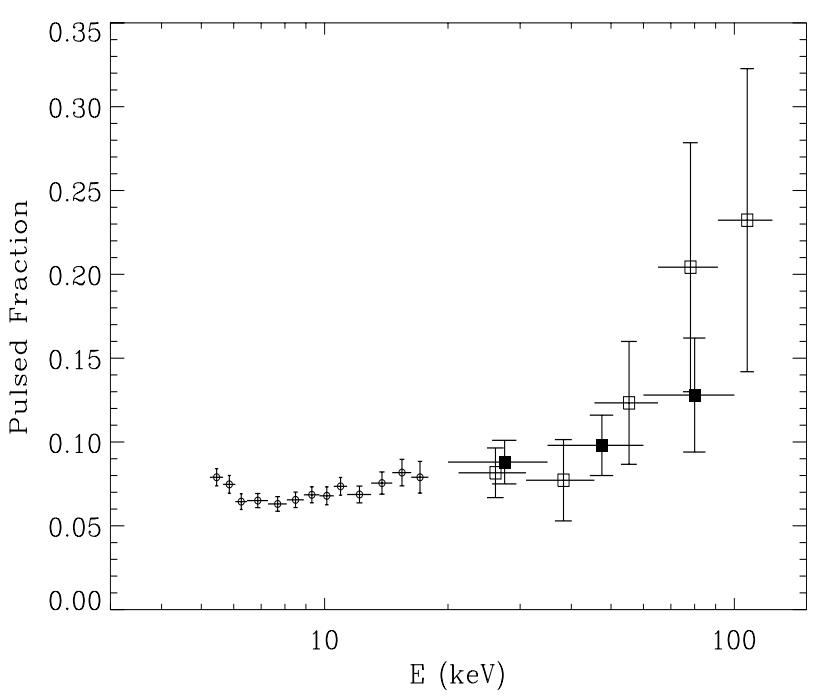

Fig. 8. The pulsed fraction (=pulsed flux/total flux) of IGR J00291+5934 using pulsed/total flux measurements from PCA/HEXTE and JEM-X/ISGRI (open circles, PCA pulsed flux relative to JEM-X total flux; open squares, HEXTE pulsed flux relative to ISGRI total flux; filled squares, ISGRI pulsed flux relative to ISGRI total flux). The pulsed fraction gradually increases from $\sim 6 \%$ at $\sim 6 \mathrm{keV}$ to $\sim 12-20 \%$ at $\sim 100 \mathrm{keV}$.

converted to flux values using (a) the pulsed excess counts of the Crab pulsar as a reference from an equivalent $5 \times 5$ on-axis dither observation (Rev-102; $54.33 \mathrm{ks}$ ), determined in the same energy bands as chosen for IGR J00291+5934, and (b) a spectral model for the pulsed emission of the Crab pulsar (see e.g. Kuiper et al. 2001) over the 15-10000 keV energy range based on HEXTE and CGRO/COMPTEL measurements. These ISGRI pulsed flux measurements are also shown in Fig. 7.

From the PCA/HEXTE and ISGRI pulsed flux measurements shown in Fig. 7 it is clear that this emission component is hardening towards higher energies. Superposing the total IGR J00291+5934 spectrum from JEM-X and ISGRI measurements (see Sect. 3.2.1) in Fig. 7, which softens weakly towards higher energies, it is clear that the pulsed fraction increases as a function of energy. The energy dependence of the pulsed fraction is shown in Fig. 8. It gradually increases from $\sim 6 \%$ at 6 $\mathrm{keV}$ to $\sim 12-20 \%$ near $100 \mathrm{keV}$. It is the first time that such behaviour has been measured for any of the known accretionpowered ms pulsars.

We note here that Galloway et al. (2005) report a decrease of the fractional rms amplitude of the pulsations with increasing energy above $10 \mathrm{keV}$. As remarked before, this can be explained by the contribution of V709 Cas in the PCA data, which has a harder spectrum than IGR J00291+5934 in this energy range.

\section{Discussion}

\subsection{Companion star and the distance}

Taking the INTEGRAL observed total fluence of $1.37 \times$

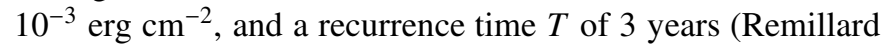
2004), we find a long term time-averaged flux of $1.45 \times$ $10^{-11} \mathrm{erg} \mathrm{cm}^{-2} \mathrm{~s}^{-1}$ (smaller than the $1.8 \times 10^{-11} \mathrm{erg} \mathrm{cm}^{-2} \mathrm{~s}^{-1}$ flux reported by Galloway et al. 2005). Following the current model of Verbunt \& van den Heuvel (1995) and Bildsten \& Chakrabarty (2001), we therefore find, for an inclination $i=90^{\circ}$ and minimum companion mass $M_{\mathrm{c}}=0.039 M_{\odot}$ (for a $1.4 M_{\odot}$ neutron star), a lower limit on the source distance of $4.7 \mathrm{kpc}$. At a most probable inclination angle $i=60^{\circ}$, we obtain a source distance of $5.4 \mathrm{kpc}$. In both cases the companion is a hot brown dwarf. The $\sim 1 \mathrm{kpc}$ difference between our calculated minimum source distance and that of Galloway et al. (2005) is attributable to the difference in observed total flux. At a distance of $\sim 5 \mathrm{kpc}$, IGR J00291+5934 lies about 270 pc off the galactic plane, placing it within the thick disk, but outside the thin disk.

\subsection{Origin of $X$-ray emission}

The spectral parameters obtained from our thermal Comptonization fits to the broad-band data for IGR J00291+5934, $k T_{\mathrm{e}}=49 \mathrm{keV}$ and $\tau_{\mathrm{T}}=1.12$, are similar to those of other MSPs: $k T_{\mathrm{e}}=60 \mathrm{keV}, \tau_{\mathrm{T}}=0.88$ in SAX J1808.4-3658, $k T_{\mathrm{e}}=33 \mathrm{keV}, \tau_{\mathrm{T}}=1.7$ in XTE J1751-350 (see Gierliński \& Poutanen 2005). We note that the product $\tau_{\mathrm{T}} \times k T_{\mathrm{e}}$ is amazingly similar for the three sources. The spectral shape and the product $\tau_{\mathrm{T}} \times k T_{\mathrm{e}}$ are very stable during the outburst as is observed in other sources too (Gilfanov et al. 1998; Gierliński \& Poutanen 2005).

The constancy of the spectral slopes during the outbursts and their extreme similarity in different MSPs can be used as an argument that the emission region geometry does not depend on the accretion rate. If the energy dissipation takes place in a hot shock, while the cooling of the electrons (that emit X/ $\gamma$-rays via thermal Comptonization) is determined by the reprocessing of the hard X-ray radiation at the neutron star surface (so called two-phase model, see Haardt \& Maraschi 1993; Stern et al. 1995; Poutanen 1998; Titarchuk et al. 1998; Malzac et al. 2001), the spectral slope is determined by the energy balance in the hot phase and is a function of the geometry. At constant geometry (e.g. slab), the temperature depends on the optical depth, but $\tau_{\mathrm{T}} \times k T_{\mathrm{e}}$ is approximately constant.

\subsection{Pulsed fraction and pulse profile}

We showed that the pulsed fraction gradually increases with energy from $\sim 6 \%$ at $6 \mathrm{keV}$ to $\sim 12-20 \%$ near $100 \mathrm{keV}$. For the first time such behaviour has been measured for any of the accretion-powered MSPs.

A spot emitting as a black body (with isotropic specific intensity) at a slowly rotating star produces nearly sinusoidal variations in the observed flux (Beloborodov 2002), and the peak is reached when the spot is closest to the observer. When the spot radiation is anisotropic, the harmonics appear in the pulse profile, with their strength depending on the degree of anisotropy (Poutanen 2004; Viironen \& Poutanen 2004). In case of rapid rotation, as in MSPs, Doppler boosting affects the variability pattern. When the spot moves towards the observer, the emission increases, while for a spot moving away, the flux drops. The Doppler factor reaches the maximum a quarter of 
the period before the peak of the projected area shifting the emission peak towards earlier phase. The observed flux due to the Doppler effect varies as the Doppler factor to the power $(3+\Gamma)$ (Poutanen \& Gierliński 2003; Viironen \& Poutanen 2004), where the photon index $\Gamma$ could be a function of energy. If the Doppler factor varies around 1 with $2 \%$ amplitude, we get $10 \%$ variability.

The Comptonized spectrum can be approximated as

$F_{E} \propto E^{-\left(\Gamma_{0}-1\right)} \exp \left(-\left[E / E_{\mathrm{c}}\right]^{\beta}\right)$,

where $E_{\mathrm{c}} \sim k T_{\mathrm{e}}$ is the energy of the cutoff and parameter $\beta \sim 2$ describes its sharpness. The local photon index is then

$\Gamma(E) \equiv 1-\frac{\mathrm{d} \ln F_{E}}{\mathrm{~d} \ln E}=\Gamma_{0}+\beta\left(E / E_{\mathrm{c}}\right)^{\beta}$.

At low energies, $\Gamma \approx \Gamma_{0}$, and rms (or pulsed fraction) is a very weak function of energy. Close to the cutoff, the spectral index rapidly increases and the pulsed fraction should grow with energy, as observed (see Fig. 8). The Comptonization models predict softening of the total spectrum with simultaneous hardening of the pulsed spectrum at higher energies.

The observed pulse profiles are almost purely sinusoidal (see Fig. 5 and also Galloway et al. 2005). The total variability amplitude is proportional to the product $\sin i \sin \theta$ (Poutanen 2004), where $\theta$ is the angular distance of the spot from rotational axis. The relative amplitude of the second harmonics to the fundamental is also proportional to the same product. The $\sim 6 \%$ observed amplitude constrains the angles $i$ and $\theta$ (see e.g. Gierliński \& Poutanen 2005). We estimate that for a $1.4 M_{\odot}$ neutron star with $12 \mathrm{~km}$ radius, $\theta$ varies between $\sim 3^{\circ}$ and $15^{\circ}$ when the inclination $i$ varies from $90^{\circ}$ to $18^{\circ}$.

\subsection{Time lags}

The observed spectrum of IGR J00291+5934 consists of a black body component from the neutron star surface and a component produced by Comptonization of these seed photons in the hot electron region, presumably a shock which can be represented as a plane-parallel slab. The angular distributions of the black body and Comptonized photons emitted by the slab are significantly different. The difference in the emission patterns causes the two components to show a different variability pattern as a function of the pulsar phase, with the hard Comptonized component leading the soft black body component (Poutanen \& Gierliński 2003; Viironen \& Poutanen 2004).

This scenario is consistent with observations of SAX J1808.4-3658 (Cui et al. 1998; Gierliński et al. 2002), XTE J1751-350 (Gierliński \& Poutanen 2005), where time lags increase rapidly with energy until $7-10 \mathrm{keV}$, where the black body contribution becomes negligible and then saturate above $10 \mathrm{keV}$. In IGR J00291+5934, however, time lags first increase until $7 \mathrm{keV}$ and then decrease slightly (see Fig. 6 and Galloway et al. 2005). Judging from the PCA data, it seems that the lags saturate above $15 \mathrm{keV}$. The decrease of the time lags above $7 \mathrm{keV}$ does not have an obvious explanation. There are also (not very significant) indications from the HEXTE and ISGRI data that the lags even reach zero value at $\sim 50 \mathrm{keV}$.
If confirmed, this kind of behaviour would be a serious challenge to any model.

\section{Summary}

We analyzed the spectral and the timing behaviour from the entire INTEGRAL observation of IGR J00291+5934, which was spatially well distinguished from the neighbouring source V709 Cas. The time averaged broad-band spectrum from 5 to $300 \mathrm{keV}$ obtained with JEM-X/ISGRI is well described by a thermal Comptonization model with seed photons from the neutron star surface scattered in a shock-heated accretion column above the hot spot region. Additionally, studying the spectral evolution, we found that the electron temperature and the related optical depth are almost invariant as the flux decreases during the outburst.

The INTEGRAL/ISGRI data, supported by simultaneously obtained $R X T E /$ PCA and HEXTE data, also allowed us to study the timing behaviour in the millisecond range for energies up to $150 \mathrm{keV}$. We detected the $1.67 \mathrm{~ms}$ period consistently with all three instruments and found that the pulse profile is close to a sine function at all studied energies as observed for the other MSPs (see e.g., Gierliński et al. 2002; Gierliński \& Poutanen 2005). This is the first time that pulsed emission has been detected from 2 up to $\sim 150 \mathrm{keV}$ for any of the currently known accretion-powered millisecond pulsars. Moreover, it demonstrates the excellent timing capabilities of INTEGRAL (see Kuiper et al. 2003) down to the $0.1 \mathrm{~ms}$ level. We have discovered that the pulsed fraction significantly increases with energy which is naturally explained by the effect of Doppler boosting on the exponentially cutoff Comptonization spectrum. We have measured a spin-up rate of $+8.4 \times 10^{-13} \mathrm{~Hz} \mathrm{~s}^{-1}$. Accurate measurements of the frequency derivative are necessary to constrain models of NS spin evolution in X-ray binaries (e.g., Lamb \& Yu 2004). For further discussion of the measurement of the spin-up rate, see Burderi et al. (2005).

From our total fluence measurements assuming that the mass accretion rate is driven by gravitational radiation, we find the minimum source distance of $4.7 \mathrm{kpc}$. This locates the source, like other accreting X-ray MSPs, within the thick disk of the Galaxy.

Acknowledgements. MF acknowledges the CNRS for financial support and thanks A. Decourche and J. Vink for the CAS A/Tycho INTEGRAL A02 data. L.K. and W.H. are supported by the Netherlands Organisation for Scientific Research (NWO), and are grateful to A. Rots, J. in 't Zand for valuable discussions. J.P. acknowledges the Academy of Finland grant 201079. M.F. and J.P. were supported in part by the NORDITA Nordic project on High Energy Astrophysics.

\section{References}

Almem, M. L., \& Wilson, J. R. 1973, ApJ, 186, 1015

Arnaud, K. A. 1996, in Astronomical Data Analysis Software and Systems V, ed. G. H. Jacoby, \& J. Barnes (San Francisco: ASP), ASP Conf. Ser., 101, 17

Beloborodov, A. M. 2002, ApJ, 566, L85

Bikmaev, I., Suleimanov, V., Galeev, A., et al. 2004, Astron. Telegram, 395 
Bildsten, L., \& Chakrabarty, D. 2001, ApJ, 557, 292

Buccheri, R., Bennett, K., Bignami, G. F., et al. 1983, A\&A, 128, 245

Burderi, L., Di Salvo, T., Lavagetto, G., et al. 2005, ApJ, submitted

Courvoisier, T. J.-L., Walter, R., Beckmann, V., et al. 2003, A\&A, 411, L57

Cui, W., Morgan, E. H., \& Titarchuk, L. G. 1998, ApJ, 504, L27

Downes, R., Webbink, R. F., \& Shara, M. M. 1997, PASP, 109, 345

Eckert, D., Walter, R., Kretschmar, P., et al. 2004, Astron. Telegram, 352

Falanga, M., Bonnet-Bidaud, J. M., Poutanen., J., et al. 2005, A\&A, 436, 647

Fender, R., De Bruyn, G., Pooley, G., \& Stappers, B. 2004, Astron. Telegram, 361

Filippenko, A. V., Foley, R. J., \& Callanan, P. J. 2004, Astron. Telegram, 366

Fox, D. B., \& Kulkarni, S. R. 2004, Astron. Telegram, 354

Galloway, D. K., Markwardt, C. B., Morgan, E. H., Chakrabarty, D., \& Strohmayer, T. E. 2005, ApJ, 622, L45

Gierliński, M., Done, C., \& Barret, D. 2002, MNRAS, 331, 141

Gierliński, M., \& Poutanen, J. 2005, MNRAS, 359, 1261

Gilfanov, M., Revnivtsev, M., Sunyaev, R., \& Churazov, E. 1998, A\&A, 338, L83

Goldwurm, A., David, P., Foschini, L., et al. 2003, A\&A, 411, L223

Gros, A., Goldwurm, A., Cadolle-Bel, M., et al. 2003, A\&A, 411, L179

Haardt, F., \& Maraschi, L. 1993, ApJ, 413, 507

Jahoda, K., Swank, J. H., Giles, A. B., et al., 1996, Proc. SPIE, 2808, 59

Jonker, P. G., Campana, S., Steeghs, D., et al. 2005, MNRAS, 361, 511

Kluz̀niak, W., \& Wilson, J. R. 1991 ApJ, 372, L87

Kuiper, L., Hermsen, W., Cusumano, G., et al. 2001, A\&A, 378, 918

Kuiper, L., Hermsen, W., Walter, R., \& Foschini, L. 2003, A\&A, 411, L31

Lamb, F. K., \& Yu, W. 2004, in Binary Radio Pulsars, ed. F. A. Rasio, \& I. H. Stairs (San Francisco: ASP), ASP Conf. Ser., in press [arXiv: astro-ph/0408459]

Lebrun, F., Leray, J.-P., Lavocate, Ph., et al. 2003, A\&A, 411, L141

Lund, N., Budtz-Joergensen, C., Westgaard, N. J., et al. 2003, A\&A, 411, L231

Lyubarskii, Yu. E., \& Sunyaev, R. A. 1982, SvA Lett., 8, 330

Malzac, J., Beloborodov, A. M., \& Poutanen, J. 2001, MNRAS, 326, 417
Manchester, R. N., \& Taylor, J. H. 1977, in Pulsars, ed. G. Burbidge, $\&$ M. Burbidge (W. H. Freeman and Company)

Markwardt, C. B., Swank, J. H., \& Strohmayer, T. E. 2004a, Astron. Telegram, 353

Markwardt, C. B., Galloway, D. K., Chakrabarty, D., Morgan, E. H., \& Strohmayer, T. E. 2004b, Astron. Telegram, 360

Mewe, R., Kaastra, J. S., \& Liedahl, D. A. 1995, Legacy J. HEASARC, 6, 16

Nowak, M. A., Paizis, A., Wilms, J., et al. 2004, Astron. Telegram, 369

Pooley, G. 2004, Astron. Telegram, 355

Poutanen, J. 1998, in Theory of Black Hole Accretion Discs, ed. M. A. Abramowicz, G. Björnsson, \& J. E. Pringle (Cambridge: Cambridge Univ. Press), 100

Poutanen, J. 2004, in X-ray Timing 2003: Rossi and Beyond, ed. P. Kaaret, F. K. Lamb, \& J. H. Swank (Melville, NY: AIP), 228

Poutanen, J., \& Gierliński, M. 2003, MNRAS, 343, 1301

Poutanen, J., \& Svensson, R. 1996, ApJ, 470, 249

Remillard, R. 2004, Astron. Telegram, 357

Roelofs, G., Jonker, P. G., Steeghs, D., Torres, M., \& Nelemans, G. 2004, Astron. Telegram, 356

Rothschild, R. E., Blanco, P. R., Gruber, D. E., et al. 1998, ApJ, 496, 538

Rupen, M. P., Dhawan, V., \& Mioduszewski, A. J. 2004, Astr. Tel., 364

Shaw, S. E., Mowlavi, N., Rodriguez, J., et al. 2005, A\&A, 432, L13

Steeghs, D., Blake, C., Bloom, J. S., et al. 2004, Astr. Tel., 363

Stern, B. E., Poutanen, J., Svensson, R., Sikora, M., \& Begelman, M. C. 1995, ApJ, 449, L13

Taylor, J. H., \& Weisberg, J. M. 1989, ApJ, 345, 434

Titarchuk, L., Lapidus, I., \& Muslimov, A. 1998, ApJ, 499, 315

Ubertini, P., Lebrun, F., Di Cocco, G., et al. 2003, A\&A, 411, L131

Verbunt, F., \& van den Heuvel, E. P. J. 1995, in X-ray binaries, ed. W. H. G. Lewin, J. van Paradijs, \& E. P. J. van den Heuvel (Cambridge: Cambridge University Press), 457

Viironen, K., \& Poutanen, J. 2004, A\&A, 426, 985

Wijnands, R. 2005, in Pulsars New Research (NY: Nova Science Publishers), in press [arXiv: astro-ph/0501264]

Winkler, C., Courvoisier, T. J.-L., Di Cocco, G., et al. 2003, A\&A, 411, L1

Zel'dovich, Ya. B., \& Shakura, N. I. 1969, Sov. Astron., 13, 175 